\title{
Calories on menus: does impact diminish with duration or flourish after festivities?
}

\author{
K. McElroy ${ }^{1}$, M. Clements ${ }^{1}$, O. Feehan ${ }^{1}$, B.E. Cronin ${ }^{1}$, B. Griffin ${ }^{1}$, F.E. Douglas ${ }^{1}$, \\ O.C. Lyons ${ }^{1,2}$ and M.A.T. Flynn ${ }^{1,2}$ \\ ${ }^{1}$ Public Health Nutrition, Food Safety Authority of Ireland, Dublin 1, Republic of Ireland and ${ }^{2}$ Northern Ireland Centre \\ for Food and Health, University of Ulster, Coleraine, BT52 1SA, UK
}

Displaying calories in food service outlets has resulted in consumers purchasing fewer calories ${ }^{(1)}$ and this effect increases with time post calorie menu labelling $(\mathrm{CML})^{(2)}$. This study examines the effect of CML in Ireland over time, taking into account the 'festive season' and gender differences.

Calories per portion of food served for breakfast (set daily menu) and lunch (variable daily menu, three week menu cycle) in a hospital staff and visitor canteen were calculated using MenuCal ${ }^{\circledR(3)}$. Consumer food choices were recorded for both meals on five days (Monday-Friday) of the same menu cycle, over four different time points - November 2014 (Pre CML), January 2015 (2 months Post CML \& Post Festive), November 2015 (12 months Post CML), January 2016 (14 months Post CML \& Post Festive). Median calories purchased by consumers at each time point, and for each meal, were compared and differences according to gender were explored. All time points were compared with Pre CML; and 2 months Post Festive was compared with 14 months Post Festive.

As shown below, compared with Pre CML median calories purchased were significantly lower at 14 months overall and for both meals separately; while at 12 months this was only apparent at lunch. Comparing the two Post Festive time points, median calories purchased were significantly lower overall and for both meals separately at 14 months vs. 2 months.

\begin{tabular}{|c|c|c|c|c|c|c|c|c|}
\hline & $(n)$ & $\begin{array}{c}\text { Pre CML } \dagger \\
\text { Median (Range) }\end{array}$ & (n) & $\begin{array}{c}2 \text { months 'Post CML \& Post Festive' } \\
\text { Median (Range) }\end{array}$ & (n) & $\begin{array}{l}12 \text { months Post CML } \\
\text { Median (Range) }\end{array}$ & (n) & $\begin{array}{c}14 \text { months 'Post CML \& Post } \\
\text { Festive' } \\
\text { Median (Range) }\end{array}$ \\
\hline Both meals (kcal) & 984 & $556(0-2700)$ & 996 & $532(2-2112)$ & 1342 & $536(0-2040)$ & 1178 & $490 * * *+(1960)$ \\
\hline Breakfast (kcal) & 517 & $512(0-2700)$ & 418 & $444(2-2112)$ & 589 & $517(0-1519)$ & 559 & $398 * * *+(0-1529)$ \\
\hline $\begin{array}{l}\text { Lunch } \\
\text { (kcal) }\end{array}$ & 467 & $628(0-1752)$ & 578 & $584(4-2109)$ & 753 & $557 * * *(0-2040)$ & 619 & $551 * * *+(0-1960)$ \\
\hline
\end{tabular}

$\dagger$ Calorie Menu Labelling. ${ }^{*} p<0 \cdot 05,{ }^{* *} p<0 \cdot 01,{ }^{* * *} p<0 \cdot 001$; significantly different from Pre CML (Mann-Whitney U).

$\$ p<0.001$; significantly different at Post Festive time points ( 2 months vs. 14 months).

Compared with Pre CML, median calories purchased at breakfast by males were significantly lower at 2 months (605 vs. $582 \mathrm{kcal}$, $p<0.05)$ and 14 months post CML $(605$ vs. 424kcal, $p<0.001)$ showing a 'Post Festive' seasonal effect. Calories purchased at breakfast by females were only significantly lower at 14 months post CML (458kcal vs. 378, $p<0.05)$. Compared with Pre CML, median calories purchased at lunch by both genders were significantly lower at the two later time-points (12 months and 14 months), and this effect was more pronounced in females (612 vs. 524 and $527 p<0.01$ females; 678 vs. 612 and 602; $p<0.05$ males - Pre CML vs. 12 months and 14 months, respectively).

In conclusion this study shows CML is associated with a reduction in calories purchased. This effect is more evident with time and may differ according to gender and festive season.

1. Green JE, Brown AG and Ohri-Vachaspati P (2014) J Acad Nutr Diet 115, 1093-1101.

2. Krieger JW, Chan NL et al. (2013) Am J Prev Med 44 (6), 595-604.

3. MenuCal ${ }^{\mathbb{R}}$. Available from: www.menucal.ie (Accessed March 2016). 